\title{
A Class of Contractions in Hilbert Space and Applications
}

\author{
by
}

\section{Nick DUNGEY}

\author{
Presented by Jerzy $Z A B C Z Y K$
}

Summary. We characterize the bounded linear operators $T$ in Hilbert space which satisfy $T=\beta I+(1-\beta) S$ where $\beta \in(0,1)$ and $S$ is a contraction. The characterizations include a quadratic form inequality, and a domination condition of the discrete semigroup $\left(T^{n}\right)_{n=1,2, \ldots}$ by the continuous semigroup $\left(e^{-t(I-T)}\right)_{t \geq 0}$. Moreover, we give a stronger quadratic form inequality which ensures that $\sup \left\{n\left\|T^{n}-T^{n+1}\right\|: n=1,2, \ldots\right\}<\infty$. The results apply to large classes of Markov operators on countable spaces or on locally compact groups.

1. Introduction. Let $\mathcal{H}$ be a complex Hilbert space. In this note, we characterize in several ways the bounded linear operators $T \in \mathcal{L}(\mathcal{H})$ which can be written in the form

$$
T=\beta I+(1-\beta) S
$$

where $S$ is a contraction $(\|S\| \leq 1)$ and $\beta \in(0,1)$. In particular, we show that $T$ has this form if and only if $T$ is a contraction which satisfies the quadratic form inequality

$$
\|f\|^{2}-\|T f\|^{2} \geq \alpha \operatorname{Re}((I-T) f, f)
$$

for some $\alpha>0$ and all $f \in \mathcal{H}$; or, if and only if the spectrum of $T$ is contained in the unit disk $\{z \in \mathbb{C}:|z| \leq 1\}$ and one has

$$
\left\|T^{n} f\right\| \leq\left\|e^{-\varepsilon n(I-T)} f\right\|
$$

for some $\varepsilon \in(0,1)$ and all $f \in \mathcal{H}, n \in \mathbb{N}:=\{1,2,3, \ldots\}$. The condition (3) is a type of domination of the discrete semigroup $\left(T^{n}\right)_{n \in \mathbb{N}}$ by the continuous time semigroup $\left(e^{-t(I-T)}\right)_{t \geq 0}$. We remark that (1) implies that the spectrum

2000 Mathematics Subject Classification: 47A30, 47A10, 60G50, 60G15.

Key words and phrases: contraction operator, Hilbert space, Markov operator, convolution operator. 
of $T$ is contained in a disk $\{z \in \mathbb{C}:|z-\beta| \leq 1-\beta\}$. The above conditions (1), (2), (3), and further conditions on $T$, are studied in Section 2 below.

For operators in Banach spaces, a generalization of (1) was considered by Nevanlinna, who obtained the following results (for details see [11, Theorem 8] and [9, Theorem 4.5.3]).

TheOREM 1.1. For $\mathcal{X}$ a complex Banach space and $T \in \mathcal{L}(\mathcal{X})$, the following two conditions are equivalent.

(I) There exist $\beta \in(0,1), S \in \mathcal{L}(X)$ such that $\sup _{n \in \mathbb{N}}\left\|S^{n}\right\|<\infty$ and $T=\beta I+(1-\beta) S$.

(II) There exist constants $c, \alpha>0$ such that

$$
\left\|e^{z T}\right\| \leq c e^{|z|\left(1-\alpha \theta^{2}\right)}
$$

for all $z \in \mathbb{C}$ with $z=|z| e^{i \theta}, \theta \in[-\pi, \pi]$.

Moreover, if these conditions hold, then

$$
\sup _{n \in \mathbb{N}}\left\|T^{n}\right\|<\infty, \quad \sup _{n \in \mathbb{N}} n^{1 / 2}\left\|T^{n}-T^{n+1}\right\|<\infty .
$$

In particular, (4) holds whenever $T$ is given by (1) with $S$ a contraction in Hilbert space. (Additional note: conversely, the author recently proved in [6] that in any Banach space, conditions (4) imply the conditions (I) and (II), and gave further conditions equivalent to these.)

The preceding results apply to very large classes of Markov operators associated with random walks, as we discuss in Section 3 below. In particular, it seems interesting that estimates of type (3) and (4) hold for many Markov operators, a result which we have not seen in the literature.

Operators satisfying the estimate $\sup _{n \in \mathbb{N}}\left(\left\|T^{n}\right\|+n\left\|T^{n}-T^{n+1}\right\|\right)<\infty$, which is stronger than (4), have recently been well studied. See [1], [2], [5], [7], [9], [10], [11, Theorem 10] and references therein. In Hilbert space, Theorem 2.3 below shows that a simple quadratic form inequality is sufficient for that estimate.

2. Proof of the main theorem. Let us fix some notation. For $a \in \mathbb{C}$, $r \geq 0$, we set $D(a ; r):=\{z \in \mathbb{C}:|z-a|<r\}, \bar{D}(a ; r):=\{z \in \mathbb{C}:|z-a| \leq r\}$ and $\overline{\mathbb{D}}:=\bar{D}(0 ; 1)=\{z \in \mathbb{C}:|z| \leq 1\}$. Let $\mathcal{H}$ be a complex Hilbert space. If $S \in \mathcal{L}(\mathcal{H})$ and if $F$ is a function holomorphic on an open neighborhood of the spectrum $\sigma(S)$, then the operator $F(S)$ is defined by the Dunford functional calculus, and the spectral mapping theorem states that $\sigma(F(S))=F(\sigma(S))$ (see [13, Section VIII.7]). Von Neumann's inequality says that if $\|S\| \leq 1$ then

$$
\|F(S)\| \leq\|F\|_{L^{\infty}(\overline{\mathbb{D}})}
$$

whenever $F$ is holomorphic on a neighborhood of $\overline{\mathbb{D}}$. 
Here is our main result.

THEOREM 2.1. Given $T \in \mathcal{L}(\mathcal{H})$, each of the following conditions (I) to (VI) is equivalent.

(I) There exists $\beta \in(0,1)$ such that $\|T-\beta I\| \leq 1-\beta$; that is, (1) holds with $\|S\| \leq 1$.

(II) $\|T\| \leq 1$, and there exists $\alpha>0$ such that (2) holds for all $f \in \mathcal{H}$.

(III) There exists $\gamma \in(0,1)$ such that $\|T f\| \leq\|\gamma f+(1-\gamma) T f\|$ for all $f \in \mathcal{H}$.

(IV) There exists $\beta \in(0,1)$ such that $\sigma(T) \subseteq \bar{D}(\beta ; 1-\beta)$ and

$$
\|F(T)\| \leq\|F\|_{L^{\infty}(\bar{D}(\beta ; 1-\beta))}
$$

for all functions $F$ which are defined and holomorphic on a neighborhood of $\bar{D}(\beta ; 1-\beta)$.

(V) $\sigma(T) \subseteq \overline{\mathbb{D}}$, and there exists $\varepsilon \in(0,1)$ such that (3) holds for all $f \in \mathcal{H}$ and $n \in \mathbb{N}$.

(VI) $\sigma(T) \subseteq \overline{\mathbb{D}}$, and there exists $\varepsilon \in(0,1)$ such that $\left\|T e^{\varepsilon(I-T)}\right\| \leq 1$.

Moreover, if these conditions hold, then $\sup _{n \in \mathbb{N}} n^{1 / 2}\left\|T^{n}-T^{n+1}\right\|<\infty$.

REMARKs. In general, given $T \in \mathcal{L}(\mathcal{H})$, the real-valued quadratic forms $Q_{T}: \mathcal{H} \rightarrow \mathbb{R}$ and $Q_{T}^{\prime}: \mathcal{H} \rightarrow \mathbb{R}$ defined by

$$
Q_{T}(f):=\|f\|^{2}-\|T f\|^{2}, \quad Q_{T}^{\prime}(f):=\operatorname{Re}((I-T) f, f)
$$

are closely related to properties of the discrete semigroup $\left(T^{n}\right)_{n \in \mathbb{N}}$ and the continuous semigroup $\left(e^{-t(I-T)}\right)_{t \geq 0}$. For example, $Q_{T} \geq 0$ if and only if $T$ is a contraction, while $Q_{T}^{\prime} \geq 0$ if and only if the semigroup $\left(e^{-t(I-T)}\right)_{t \geq 0}$ is contractive. From this point of view, the equivalence of conditions (II) and $(\mathrm{V})$ above is not so surprising.

Note that $Q_{T} \leq 2 Q_{T}^{\prime}$ for arbitrary $T \in \mathcal{L}(\mathcal{H})$; this result is a consequence of the identity

$$
\|f\|^{2}-\|T f\|^{2}+\|(I-T) f\|^{2}=2 \operatorname{Re}((I-T) f, f)
$$

valid for all $f \in \mathcal{H}$. Observe also that there exist non-contractions $T$ satisfying $Q_{T} \geq \alpha Q_{T}^{\prime}$ for some $\alpha>0$; for example, take $T=2 I, \alpha=3$.

Proof of Theorem 2.1. The statement that $\sup _{n} n^{1 / 2}\left\|T^{n}-T^{n+1}\right\|<\infty$ is a consequence of condition (I) and Theorem 1.1. Alternatively, this final statement can be derived by applying condition (IV) to the functions $z \mapsto$ $(1-z) z^{n}$.

Condition (I) means that $\|T f-\beta f\|^{2} \leq(1-\beta)^{2}\|f\|^{2}$ for all $f \in \mathcal{H}$. By expanding

$$
\|T f-\beta f\|^{2}=\|T f\|^{2}-2 \beta \operatorname{Re}(T f, f)+\beta^{2}\|f\|^{2}
$$


and rearranging, we see that condition (I) is equivalent to the inequality

$$
\begin{aligned}
2 \beta \operatorname{Re}((I-T) f, f) & \leq(1-\beta)^{2}\|f\|^{2}+2 \beta\|f\|^{2}-\beta^{2}\|f\|^{2}-\|T f\|^{2} \\
& =\|f\|^{2}-\|T f\|^{2} .
\end{aligned}
$$

Thus condition (I) implies (II) with $\alpha=2 \beta$. Conversely, assume that condition (II) holds; then $\|T\| \leq 1$ implies that $\operatorname{Re}((I-T) f, f) \geq 0$, and hence by decreasing $\alpha$ if necessary we can assume that $\alpha \in(0,2)$. Then condition (I) holds with $\beta=\alpha / 2$.

Next, condition (III) implies that $\|T f\| \leq \gamma\|f\|+(1-\gamma)\|T f\|$, so that $\|T\| \leq 1$. Moreover, by squaring and expanding one rewrites condition (III) as

$$
\|T f\|^{2} \leq \gamma^{2}\|f\|^{2}+(1-\gamma)^{2}\|T f\|^{2}+2 \gamma(1-\gamma) \operatorname{Re}(T f, f)
$$

or equivalently, after rearranging,

$$
2(1-\gamma)(2-\gamma)^{-1} \operatorname{Re}((I-T) f, f) \leq\|f\|^{2}-\|T f\|^{2}
$$

for all $f \in \mathcal{H}$. From this, it is easy to deduce that conditions (III) and (II) are equivalent.

Let us derive condition (IV) from condition (I). One has

$$
\sigma(T)=\beta+\sigma(T-\beta I) \subseteq \beta+\bar{D}(0 ; 1-\beta)=\bar{D}(\beta ; 1-\beta)
$$

by condition (I). The desired estimate of $\|F(T)\|$ is easily obtained by applying von Neumann's inequality to the contraction $S:=(1-\beta)^{-1}(T-\beta I)$.

Conversely, condition (IV) implies (I) trivially, by considering the function $F(z)=z-\beta$.

Conditions $(\mathrm{V})$ and $(\mathrm{VI})$ are easily seen to be equivalent, since $e^{\varepsilon(I-T)}$ is the operator inverse to $e^{-\varepsilon(I-T)}$.

To show that condition (IV) implies (VI) with $\varepsilon=\beta$, we check that the holomorphic function

$$
F_{\beta}(z):=z e^{\beta(1-z)}
$$

satisfies $\left\|F_{\beta}\right\|_{L^{\infty}(\bar{D}(\beta ; 1-\beta))} \leq 1$. For $z \in \bar{D}(\beta ; 1-\beta)$, the inequality $|z-\beta|^{2} \leq$ $(1-\beta)^{2}$ implies that

$$
|z|^{2} \leq 1-2 \beta(1-\operatorname{Re}(z)) \leq e^{-2 \beta(1-\operatorname{Re}(z))}=\left|e^{-\beta(1-z)}\right|^{2}
$$

by the elementary estimate $1-t \leq e^{-t}, t \in \mathbb{R}$. Thus $\left|F_{\beta}(z)\right| \leq 1$, and $\left\|F_{\beta}\right\|_{L^{\infty}(\bar{D}(\beta ; 1-\beta))} \leq 1$.

Finally, we show that condition (VI) implies (I). This proof can be compared with [7, Section 2], where the inverse of the mapping $z \mapsto z e^{z}$ is used for a different purpose. Take $\varepsilon \in(0,1)$ as in condition (VI), and write $F_{\varepsilon}(z)=z e^{\varepsilon(1-z)}, z \in \mathbb{C}$. We need the following results on $F_{\varepsilon}$.

Lemma 2.2. Given $\varepsilon \in(0,1)$, put $A_{\varepsilon}:=\left\{z \in \mathbb{C}:|z| \leq 1,\left|F_{\varepsilon}(z)\right| \leq 1\right\}$. There exists a holomorphic function $W_{\varepsilon}$ defined on a neighborhood of $\overline{\mathbb{D}}$ such 
that $W_{\varepsilon}\left(F_{\varepsilon}(z)\right)=z$ for all $z$ in some neighborhood of $A_{\varepsilon}$. Moreover, there exists a $\beta=\beta(\varepsilon) \in(0, \varepsilon)$ such that $\bar{D}(\varepsilon ; 1-\varepsilon) \subseteq A_{\varepsilon} \subseteq \bar{D}(\beta ; 1-\beta)$ and

$$
W_{\varepsilon}(\overline{\mathbb{D}}) \subseteq \bar{D}(\beta ; 1-\beta) \text {. }
$$

Proof of Lemma 2.2. There exists a holomorphic function

$$
\widetilde{W}: D\left(0 ; e^{-1}\right) \rightarrow \mathbb{C}
$$

such that $\widetilde{W}\left(z e^{-z}\right)=z$ for all $z$ in some neighborhood of 0 ; see, for example, [7, p. 465]. Then defining $W_{\varepsilon}: D\left(0 ; \varepsilon^{-1} e^{\varepsilon-1}\right) \rightarrow \mathbb{C}$ by

$$
W_{\varepsilon}(w):=\varepsilon^{-1} \widetilde{W}\left(\varepsilon e^{-\varepsilon} w\right),
$$

one has $W_{\varepsilon}\left(F_{\varepsilon}(z)\right)=z$ for all $z$ in a neighborhood of 0 . Because $\varepsilon^{-1} e^{\varepsilon-1}>1$, the domain of definition of $W_{\varepsilon}$ contains $\overline{\mathbb{D}}$. After observing that $A_{\varepsilon}$ is a compact connected region containing 0 , one sees by analytic continuation that $W_{\varepsilon}\left(F_{\varepsilon}(z)\right)=z$ for all $z$ in a neighborhood of $A_{\varepsilon}$.

It is not difficult to show that $F_{\varepsilon}$ maps $A_{\varepsilon}$ bijectively onto $\overline{\mathbb{D}}$, and that $W_{\varepsilon}(\overline{\mathbb{D}})=A_{\varepsilon}$. The inclusion $\bar{D}(\varepsilon ; 1-\varepsilon) \subseteq A_{\varepsilon}$ was established in the argument that (IV) implies (VI).

To complete the proof of the lemma, we show that $A_{\varepsilon} \subseteq \bar{D}(\beta ; 1-\beta)$ for some $\beta \in(0, \varepsilon)$. Fixing $c \in(0,1)$ such that $e^{-t} \leq 1-c t$ for all $t \in[0,4]$, we have

$$
|z|^{2} \leq e^{-2 \varepsilon(1-\operatorname{Re}(z))} \leq 1-2 c \varepsilon(1-\operatorname{Re}(z))
$$

for all $z \in A_{\varepsilon}$. Thus $|z-c \varepsilon|^{2} \leq(1-c \varepsilon)^{2}$, and $A_{\varepsilon} \subseteq \bar{D}(c \varepsilon ; 1-c \varepsilon)$.

Continuing the notation of Lemma 2.2, assume that $T$ satisfies condition (VI). Then $\sigma(T) \subseteq \overline{\mathbb{D}}$ and, by the spectral mapping theorem, $F_{\varepsilon}(\sigma(T))=$ $\sigma\left(T e^{\varepsilon(I-T)}\right) \subseteq \overline{\mathbb{D}}$. Therefore $\sigma(T) \subseteq A_{\varepsilon}$. By Lemma 2.2 and the Dunford functional calculus we may write

$$
T-\beta I=W_{\varepsilon}\left(T e^{\varepsilon(I-T)}\right)-\beta I .
$$

The hypothesis $\left\|T e^{\varepsilon(I-T)}\right\| \leq 1$ and von Neumann's inequality then yield

$$
\|T-\beta I\| \leq \sup _{z \in \mathbb{D}}\left|W_{\varepsilon}(z)-\beta\right| \leq 1-\beta
$$

with the last inequality by (6). This establishes condition (I), and the proof of Theorem 2.1 is complete.

ExAmple. For a normal operator $T \in \mathcal{L}(\mathcal{H})$, it is easy to see that the conditions of Theorem 2.1 hold if and only if $\sigma(T) \subseteq \bar{D}(\beta ; 1-\beta)$ for some $\beta \in(0,1)$. For self-adjoint $T$, the conditions hold if and only if $\sigma(T) \subseteq(-1,1]$ (and in that case, the inequality (7) below is also satisfied).

To conclude this section, we describe a quadratic form inequality which is stronger than (2) and implies that $\sup _{n \in \mathbb{N}} n\left\|T^{n}-T^{n+1}\right\|<\infty$. 
Theorem 2.3. Let $T \in \mathcal{L}(\mathcal{H})$ be such that there exists $\alpha>0$ with

$$
\|f\|^{2}-\|T f\|^{2} \geq \alpha|((I-T) f, f)|
$$

for all $f \in \mathcal{H}$. Then the conditions of Theorem 2.1 are satisfied, and moreover $\sup _{n \in \mathbb{N}} n\left\|T^{n}-T^{n+1}\right\|<\infty$.

Proof. Inequality (7) implies that $\|f\|^{2}-\|T f\|^{2} \geq 0$ so that $T$ is a contraction. Thus (7) implies condition (II) of Theorem 2.1. Also, (7) and (5) show that

$$
|((I-T) f, f)| \leq 2 \alpha^{-1} \operatorname{Re}((I-T) f, f) .
$$

This last inequality is a sectorial estimate which implies (see for example $[8$, Theorem IX.1.24]) that the semigroup $\left(e^{-t(I-T)}\right)_{t \geq 0}$ is bounded holomorphic, hence one has an estimate $\left\|(I-T) e^{-t(I-T)}\right\| \leq c t^{-1}$ for all $t>0$. Then using (3) yields a bound

$$
\left\|(I-T) T^{n}\right\| \leq\left\|(I-T) e^{-\varepsilon n(I-T)}\right\| \leq c^{\prime} n^{-1}
$$

for all $n \in \mathbb{N}$.

In connection with Theorem 2.3 and its proof, we mention the situation in general Banach spaces. For a bounded linear operator in Banach space, the condition $\sup _{n \in \mathbb{N}}\left(\left\|T^{n}\right\|+n\left\|T^{n}-T^{n+1}\right\|\right)<\infty$ holds if and only if $\sigma(T) \subseteq D(0 ; 1) \cup\{1\}$ and the semigroup $\left(e^{-t(I-T)}\right)_{t \geq 0}$ is bounded holomorphic (see [10, Theorem 2.1] and [1,2]). The simple proof above of Theorem 2.3 depended crucially on the estimate (3).

3. Markov operators. This section presents two distinct settings of Markov operators where the preceding results apply.

Markov operators on a countable space. Let $T$ be a Markov operator on a countable set $\Omega$. That is, we assume that there exists a Markov kernel $(p(x, y))_{x, y \in \Omega}$ with $p(x, y) \geq 0, \sum_{y \in \Omega} p(x, y)=1$, such that $T$ acts on bounded functions $f: \Omega \rightarrow \mathbb{C}$ by the formula

$$
(T f)(x)=\sum_{y \in \Omega} p(x, y) f(y)
$$

for all $x \in \Omega$. Suppose further that $m$ is an invariant measure for $p$, meaning that $m: \Omega \rightarrow(0, \infty)$ with $\sum_{x \in \Omega} m(x) p(x, y)=m(y)$ for all $y \in \Omega$. For subsets $A \subseteq \Omega$ we set $m(A):=\sum_{x \in A} m(x)$. Then it is standard that $T$ is a contraction in the Banach spaces $L^{r}(\Omega ; m), r \in[1, \infty]$.

Our result in this setting is the following.

Proposition 3.1. Suppose there exists $\beta \in(0,1)$ such that

$$
p(x, x) \geq \beta
$$


for all $x \in \Omega$. Then $T$ satisfies the conditions of Theorem 1.1 in each of the Banach spaces $L^{r}(\Omega ; m), r \in[1, \infty]$, and satisfies the conditions of Theorem 2.1 in the Hilbert space $L^{2}(\Omega ; m)$.

Proof. Observe from (8) that the operator $S:=(1-\beta)^{-1}(T-\beta I)$ is also a Markov operator, corresponding to the Markov kernel $q(x, y):=(1-$ $\beta)^{-1}\left(p(x, y)-\beta \delta_{x}(y)\right)$ (where $\delta_{x}(y)$ is 1 or 0 according as $x=y$ or $x \neq y$ ). The measure $m$ is invariant for $q$. Thus $S$ is a contraction in $L^{r}(\Omega ; m)$, $r \in[1, \infty]$, and the proposition follows from Theorems 1.1 and 2.1.

The condition (8) is not new and has been used in studying random walks; see, for example, [3] and references therein, and see also [4, Lemma 1.3] for a result essentially weaker than Proposition 3.1. While many authors assume that $p$ is reversible with respect to $m$, which implies that $T$ is self-adjoint in $L^{2}(\Omega ; m)$, we make no reversibility assumption above.

Markov convolution operators on a locally compact group. Let $G$ be a locally compact, compactly generated group, with a fixed left invariant Haar measure $d g$. (For background information, see [12] and [5].) We fix an open, relatively compact neighborhood $U$ of the identity $e$ of $G$ such that $U=U^{-1}$ and $U$ generates $G$. The modulus $\varrho=\varrho_{U}: G \rightarrow \mathbb{N}$ is defined by $\varrho(g):=$ $\inf \left\{n \in \mathbb{N}: g \in U^{n}\right\}$, where $U^{n} \subseteq G$ denotes the set of all $n$-fold products of elements of $U$.

Given $\mu \in \mathbb{P}(G)$, where $\mathbb{P}(G)$ is the set of regular Borel probability measures on $G$, consider the Markov convolution operator $T_{\mu}$ given by $T_{\mu} f:=$ $\mu * f$ for all $f \in L^{r}:=L^{r}(G ; d g), r \in[1, \infty]$. The involute $\mu^{*} \in \mathbb{P}(G)$ of $\mu$ is defined by $\mu^{*}(A):=\mu\left(A^{-1}\right)$ for Borel sets $A \subseteq G$. We say that $\mu$ is adapted if the smallest closed subgroup of $G$ containing the support of $\mu$ is $G$ itself; alternatively, say that $\mu$ is aperiodic if the measure $\mu^{*} * \mu$ is adapted. Aperiodicity is a stronger condition than adaptedness.

Here is our result in this setting.

TheOREM 3.2. Let $\mu \in \mathbb{P}(G)$ be aperiodic, non-singular with respect to Haar measure $d g$, and such that $\int_{G} d \mu(g) \varrho(g)^{2}<\infty$. Then $T_{\mu}$ satisfies the conditions of Theorem 2.1 in the Hilbert space $L^{2}=L^{2}(G ; d g)$.

In the situation of Theorem 3.2, unlike that of Proposition 3.1, the operator $S:=(1-\beta)^{-1}\left(T_{\mu}-\beta I\right)$ is not necessarily a Markov operator for any $\beta \in(0,1)$. For example, consider the discrete group $G=\mathbb{Z}$ of integers and an aperiodic $\mu \in \mathbb{P}(\mathbb{Z})$ which satisfies $\mu(\{0\})=0$.

The proof of Theorem 3.2 requires the following lemma which is contained in, for example, [5, Propositions 3.2, 3.3]; results in the same spirit are in [12, Chapters VI, VII].

Lemma 3.3. Suppose that $\nu \in \mathbb{P}(G)$ is symmetric (that is, $\nu^{*}=\nu$ ), adapted, non-singular with respect to $d g$, and such that $\int_{G} d \nu(g) \varrho(g)^{2}<\infty$. 
Write $\Gamma(f):=\int_{U} d g \int_{G} d h|f(g h)-f(h)|^{2}$. Then there exists $c>1$ such that

$$
c^{-1} \Gamma(f) \leq\left(\left(I-T_{\nu}\right) f, f\right) \leq c \Gamma(f)
$$

for all $f \in L^{2}$.

Proof of Theorem 3.2. The measures $\nu_{1}:=2^{-1}\left(\mu+\mu^{*}\right) \in \mathbb{P}(G)$ and $\nu_{2}:=\mu^{*} * \mu \in \mathbb{P}(G)$ satisfy the hypotheses of Lemma 3.3. Since $T_{\mu^{*}}$ is the adjoint operator of $T_{\mu}$ in $L^{2}$, one finds that

$$
\begin{aligned}
\operatorname{Re}\left(\left(I-T_{\mu}\right) f, f\right) & =2^{-1}\left(\left(I-T_{\mu}\right) f, f\right)+2^{-1}\left(f,\left(I-T_{\mu}\right) f\right) \\
& =\left(\left(I-T_{\nu_{1}}\right) f, f\right) \\
& \leq c \Gamma(f) \\
& \leq c^{\prime}\left(\left(I-T_{\nu_{2}}\right) f, f\right)=c^{\prime}\left(\|f\|_{2}^{2}-\left\|T_{\mu} f\right\|_{2}^{2}\right)
\end{aligned}
$$

for all $f \in L^{2}$. Thus condition (II) of Theorem 2.1 is satisfied.

More refined $L^{2}$ estimates depend on the notion of centeredness for probability measures; see [5] for details. Actually, if $\mu \in \mathbb{P}(G)$ is as in Theorem 3.2 and is centered, then [5, Proposition 3.2] gives an estimate $\left|\left(\left(I-T_{\mu}\right) f, f\right)\right| \leq c \Gamma(f), f \in L^{2}$, so that $T_{\mu}$ satisfies the inequality $(7)$.

\section{References}

[1] S. Blunck, Perturbation of analytic operators and temporal regularity of discrete heat kernels, Colloq. Math. 86 (2000), 189-201.

[2] -, Analyticity and discrete maximal regularity on $L_{p}$-spaces, J. Funct. Anal. 183 (2001), 211-230.

[3] T. Coulhon, A. Grigor'yan and F. Zucca, The discrete integral maximum principle and its applications, Tohoku Math. J. 57 (2005), 559-587.

[4] N. Dungey, A note on time regularity for discrete time heat kernels, Semigroup Forum 72 (2006), 404-410.

[5] - Time regularity for random walks on locally compact groups, Probab. Theory Related Fields 137 (2007), 429-442.

[6] -, On time regularity and related conditions for power-bounded operators, Proc. London Math. Soc., to appear.

[7] N. Kalton, S. Montgomery-Smith, K. Oleszkiewicz and Y. Tomilov, Power-bounded operators and related norm estimates, J. London Math. Soc. 70 (2004), 463-478.

[8] T. Kato, Perturbation Theory for Linear Operators, 2nd ed., Grundlehren Math. Wiss. 132, Springer, 1980.

[9] O. Nevanlinna, Convergence of Iterations for Linear Equations, Birkhäuser, Basel, 1993.

[10] -, On the growth of the resolvent operators for power-bounded operators, in: Linear Operators, J. Janas et al. (eds.), Banach Center Publ. 38, Inst. Math., Polish Acad. Sci., 1997, 247-264.

[11] —, Resolvent conditions and powers of operators, Studia Math. 145 (2001), 113-134.

[12] N. T. Varopoulos, L. Saloff-Coste and T. Coulhon, Analysis and Geometry on Groups, Cambridge Tracts in Math. 100, Cambridge Univ. Press, 1992. 
[13] K. Yosida, Functional Analysis, 6th ed., Grundlehren Math. Wiss. 123, Springer, 1980.

Nick Dungey

Department of Mathematics

Macquarie University

Sydney, NSW 2109

Australia

E-mail: ndungey@ics.mq.edu.au 